\title{
Shock Scattering in a Multiphase Flow Model
}

\author{
D. E. Klem
}

April 8, 2003

U.S. Department of Energy

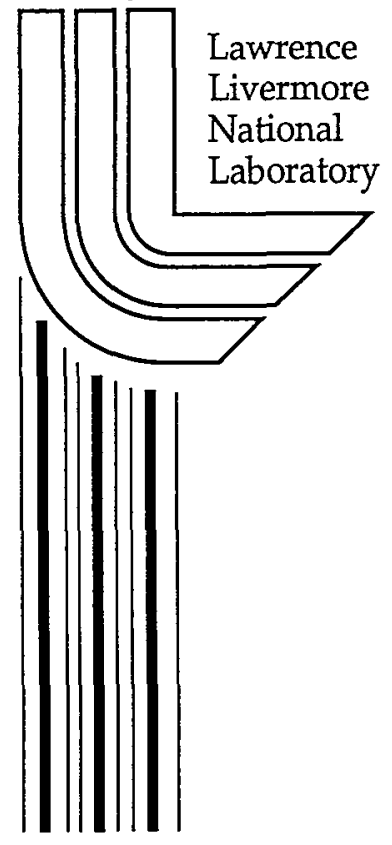




\section{DISCLAIMER}

This document was prepared as an account of work sponsored by an agency of the United States Government. Neither the United States Government nor the University of California nor any of their employees, makes any warranty, express or implied, or assumes any legal liability or responsibility for the accuracy, completeness, or usefulness of any information, apparatus, product, or process disclosed, or represents that its use would not infringe privately owned rights. Reference herein to any specific commercial product, process, or service by trade name, trademark, manufacturer, or otherwise, does not necessarily constitute or imply its endorsement, recommendation, or favoring by the United States Government or the University of California. The views and opinions of authors expressed herein do not necessarily state or reflect those of the United States Government or the University of California, and shall not be used for advertising or product endorsement purposes.

This work was performed under the auspices of the U. S. Department of Energy by the University of California, Lawrence Livermore National Laboratory under Contract No. W-7405-Eng-48.

This report has been reproduced directly from the best available copy.

Available electronically at http://www.doc.gov/bridge

Available for a processing fee to U.S. Department of Energy

And its contractors in paper from

U.S. Department of Energy

Office of Scientific and Technical Information

P.O. Box 62

Oak Ridge, TN 37831-0062

Telephone: (865) 576-8401

Facsimile: (865) 576-5728

E-mail: reports@adonis.osti.gov

Available for the sale to the public from

U.S. Department of Commerce

National Technical Information Service

5285 Port Royal Road

Springfield, VA 22161

Telephone: (800) 553-6847

Facsimile: (703) 605-6900

E-mail: orders@ntis.fedworld.gov

Online ordering: http://www.ntis.gov/ordering.htm

OR

Lawrence Livermore National Laboratory

Technical Information Department's Digital Library

http://www.llnl.gov/tid/Library.html 


\title{
Shock Scattering in a Multiphase Flow Model
}

\author{
Daniel E. Klem \\ UCRL-XX-YYYYYY
}

\begin{abstract}
Multiphase flow models have been proposed for use in situations which have combined Rayleigh-Taylor (RTI) and Richtmyer-Meshkov (RMI) instabilities[2, 3]. Such an approach works poorly for the case of a heavy to light shock incidence on a developed interface. The physical original of this difficulty is traced to an inadequate model of the interfacial pressure term as it appears in the momentum and turbulence kinetic energy equations. Constraints on the form of a better model from a variety of sources are considered. In this context it is observed that a new constraint on closures arises. This occurs because of the discontinuity within the shock responsible for the RMI. The proposed model (Shock Scattering) is shown to give useful results.
\end{abstract}

\section{Introduction}

Multiphase flow models have been proposed for use in situations which have combined Rayleigh-Taylor (RTI) and Richtmyer-Meshkov (RMI) instabilities[2, 3]. Such an approach works poorly for the case of a heavy to light shock incidence on a developed interface. This occurs because of an inadequacy in the modeling of the interfacial pressures. The situation is illustrated in Fig. 1. A shock interacting with a complex surface produces transmitted and reflected waves which cannot be represented by the mean flow $\tilde{u}_{i}$. Note that this difference $u_{i}^{\prime \prime}=u_{i}-\tilde{u}_{i}$ between the actual complex velocity $u_{i}$, and the mean flow $\tilde{u}_{i}$ arises from a shock-mix interaction, and not from a shock-turbulence interaction. I.e., initially $u_{i}^{\prime \prime}=0$.

In most models fluid motions which cannot be represented by $\tilde{u}_{i}$ are absorbed into a quantity like $k=\frac{1}{2 \bar{\rho}} \cdot \overline{\rho u_{i}^{\prime \prime} u_{i}^{\prime \prime}}{ }^{1}$ If this is the case, then we should expect a source term in the time evolution equation for $k$ of the form

$$
\frac{\partial}{\partial t}(\rho k)+\cdots=\cdots+E^{\prime}(\text { shocks, } \operatorname{mix})
$$

\footnotetext{
${ }^{1}$ This is the simplest case. One might also have multiple $k$ 's for the small and large scales, direction specific $k$ 's to capture anisotropies in the flow, etc.
} 

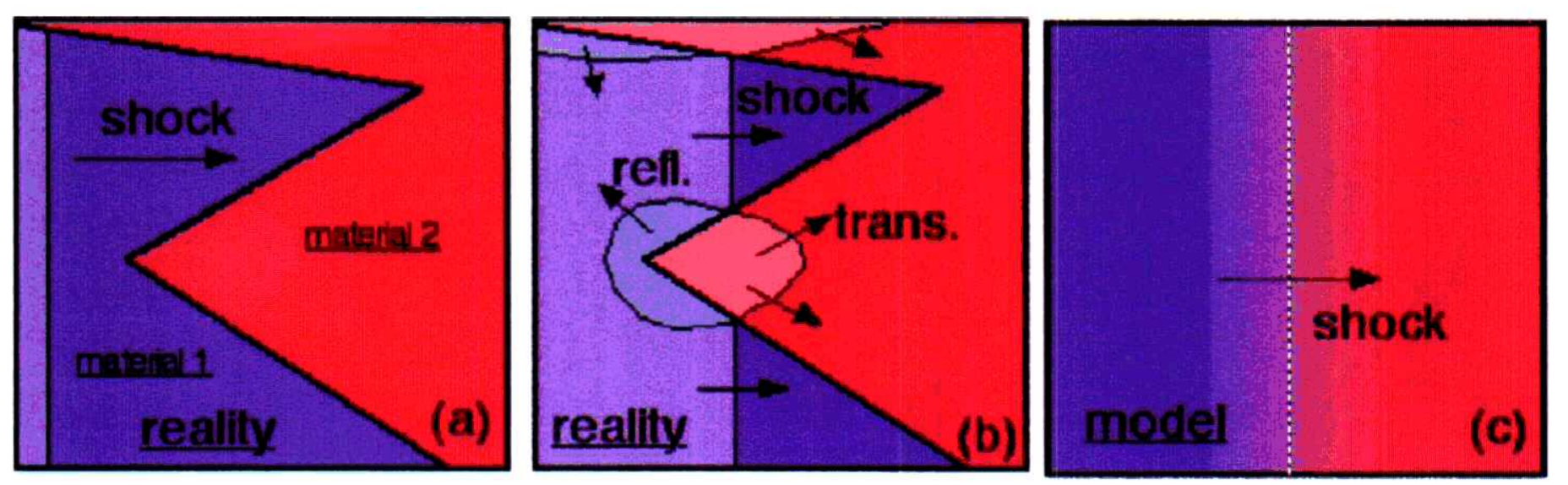

Figure 1: The figure shows a very schematic representation of the interaction of a shock with a mixed region. Part (a) is before the shock (incident from the left) interacts with the interface. Part (b) shows the interaction of the shock with the interface and the generation complex transmitted and reflected waves. Part (c) shows the view of this by the mix model.

where $E^{\prime}$ depends on the presence of a shock and a mixed region, but not on the presence of velocity fluctuations. I.e., a term which does not vanish just because $k=0$. Such a term arises naturally in the context of a multiphase flow model, and I propose that it be called "shock scattering."

The balance of this paper will consist of 1) a motivation for the use of multiphase flow models, 2) a description of how interfacial pressure terms arise, 3) consideration of the constraints on the modeling of these terms, 4) a description of an implementation of the model, and 5) a comparison to a couple of experiments.

\section{Motivation: Mix is not just turbulence}

Because this work relies heavily on the formalism used to derive the equations of multiphase flow[1], it is useful to review this so as to appreciate its applicability to the case of mixing ${ }^{2}$ produced by a combination of the Rayleigh-Taylor and RichtmyerMeshkov instabilities. In the presence of $n$ materials or phases ${ }^{3}$ the mass or continuity equation must be split into $n$ separate equations, one for each phase- $r$ :

$$
\frac{\partial \rho_{r}}{\partial t}+\frac{\partial}{\partial x_{i}}\left(\rho_{r} u_{i}\right)=0, \quad \rho=\sum_{r} \rho_{r}
$$

(The index $i$ labels a direction and the index $r$ labels a phase.) Note that this is simply the physics and not a modeling equation. It is valid at every point in space

\footnotetext{
${ }^{2}$ In this paper I am going to use the term "mix" to mean the interpenetration of two (or more) fluids. The subsequent molecular mix is not considered.

${ }^{3}$ I am going to use the term "phase" in the balance of this paper only because it has been used in the past. The reader might just as well understand this as "material" or "component."
} 
(trivially if the phase is not present). It contains only a single velocity $u_{i}$. This increase in complexity is required for the simple reason that one now needs to keep track of the locations of $n$ different materials. Associated with this is a single (vector) momentum equation

$$
\frac{\partial}{\partial t}\left(\rho u_{i}\right)+\frac{\partial}{\partial x_{j}}\left(\rho u_{j} u_{i}\right)=-\frac{\partial p}{\partial x_{i}}
$$

and an analogous equation for the internal energy.

The need for a model arises when the complexity of the flow makes the calculation of details of the interpenetration (mix) impractical or unnecessary. In such a case it is hopefully not necessary to know $\rho_{r}$ at every point in space and time, but rather it is sufficient to know only an average value $\overline{\rho_{r}}$. The bar here denotes an averaging operation which is to be understood in either an ensemble or (maybe) a volume sense. See reference [1]. The equation for the averaged quantity is obtained almost trivially

$$
\frac{\partial \overline{\rho_{r}}}{\partial t}+\frac{\partial}{\partial x_{i}}\left(\overline{\rho_{r} u_{i}}\right)=0
$$

where the second term illustrates the problem. This term contains an unknown correlation between the presence of the phase- $r\left(\rho_{r}\right)$ and the velocity $\left(u_{i}\right)$. Hence the problem of mix modeling reduces to that of modeling the correlation $\overline{\rho_{r} u_{i}}$.

As an aside it is useful to consider a path commonly taken in mix modeling. One can define both an average density $\bar{\rho}$ and a mass weighted average velocity $\tilde{u}_{i}=\overline{\rho u_{i}} / \bar{\rho}$ without regard to the presence of absence of a particular phase. From this one can define a velocity fluctuation $u_{i}^{\prime \prime}=u_{i}-\tilde{u}_{i}$ which makes it possible to write the previous equation as

$$
\frac{\partial \overline{\rho_{r}}}{\partial t}+\frac{\partial}{\partial x_{i}}\left(\bar{\rho}_{r} \tilde{u}_{i}\right)=-\frac{\partial}{\partial x_{i}}\left(\overline{\rho_{r} u_{i}^{\prime \prime}}\right)
$$

Since the advection term on the left-hand side contains the same velocity for every phase, this term will not produce any mixing. The problem then reduces to modeling the correlation in the term on the right side $\overline{\rho_{r} u_{i}^{\prime \prime}}$. At this point it is not uncommon to concede defeat by invoking a gradient diffusion closure. I.e., taking

$$
\overline{\rho_{r} u_{i}^{\prime \prime}}=-D \frac{\partial \overline{\rho_{r}}}{\partial x}
$$

where $D \propto \sqrt{k} l$, for instance. While a closure such as this has a very definite practical advantages in its implementation, its utility for modeling instability driven mix is unproven. Given that it has its motivation in the ideas of isotropic turbulence and analogies to kinetic theory, it is not obvious that such a closure should be applicable to the present problem. Such closures are often accompanied by complicated turbulence 
turbulent mixing layer was observed to first grow, then demix during the deceleration, and then resume growing during the final coast phase. This subsequent regrowth is interpreted as being due to the turbulence[2].

The two fluids here (actually a calcium chloride solution and hexane, densities of $1.142 \mathrm{~g} / \mathrm{cm}^{2}$ and $0.66 \mathrm{~g} / \mathrm{cm}^{2}$ ) are modeled with a scaled a ideal gas equation-of-state. The initial temperature distribution through the problem is set to produce the pressure gradient required to give a uniform initial acceleration. Subsequent changes in the acceleration are implemented through changes to the external pressure boundary conditions and necessarily produce a series of small shocks (below the threshold for the shock finder). The results of the simulation are compared to the experiment in Fig. 4. Reasonable agreement is obtained.

This is a significant experiment because it tests not only the multiphase flow part of the model, but also the $k l$ turbulence part. A substantial effort was devoted to finding parameters which would simultaneously fit both this experiment and the reshock experiment. The nature of the difficulty is as follows. The length scale $l$ is a sort of quasi-conserved quantity. I.e., just because one would like more $\int l d x$, does not mean the terms on the right-hand side will produce it. In order to grow the mix region, the model must increase $\int l d x$. In the case of the RTI, the $S_{l}$ term generates the required $l$. In the current case the $S_{l 2}$ term was added to enable the growth of the mixing region in the reshock experiment. It does this by allowing turbulence to generate $l$ after the passage of the shock. While it appears possible to match the Vetter experiment with a variety of values of $c_{10}$ and $c_{l s c 2}$ (they are anti-correlated), not perturbing the acceleration / deceleration experiment is more difficult. This sets an upper limit on the value of $c_{l s c 2}$ (larger values produce too much mix at late times). The resulting value of $c_{10}=4$ appears large in that it implies that the energy removed from the main flow and moved into $k$ is about the same size as that moved into internal energy. ${ }^{9}$ If the change in $k$ produced by the passage of the shock is, in fact, too big, then another mechanism for producing $l$ may be required.

\section{Conclusions}

This paper has proposed an innovative method to improve the behaviour of an existing model for the mix from combined RMI and RTI. The added terms improve the modeling of the interfacial pressures. Reasonable agreement is obtained with two experiments; one RM and the other RT. Additional comparisons to other mix experiments are required. A further examination of the modifications to the $l$ equation is planned.

\footnotetext{
${ }^{9}$ Recall that $\sum_{r} f_{r} H_{r, i}$ can not be greater than $\frac{1}{4}$ At.
} 
models (perhaps to obscure the fact that the proponent gave up on the initial $\overline{\rho_{r} u_{i}^{\prime \prime}}$ term).

In any case a more reasonable path is to model the mass flux in question $\overline{\rho_{r} u_{i}}$ and then use it to define a phase specific mass averaged velocity $\tilde{u}_{r, i}={\overline{\rho_{r}}}_{u_{i}} / \bar{\rho}_{r}$. This results in a set of phase specific mass conservation equations (i.e., multiphase flow equations)

$$
\frac{\partial \overline{\rho_{r}}}{\partial t}+\frac{\partial}{\partial x_{i}}\left(\bar{\rho}_{r} \tilde{u}_{r, i}\right)=0
$$

Note that this procedure has closed these equations without introducing a closure approximation as such.

This is a cute trick if one can produce an evolution equation for $\tilde{u}_{r, i}$. Such an equation can, in fact, be obtained as follows. If the momentum equation is valid at every point in space, then it is also valid in the phase-r. I.e.,

$$
\underbrace{\frac{\partial}{\partial t}\left(\rho u_{i}\right)+\frac{\partial}{\partial x_{j}}\left(\rho u_{j} u_{i}\right)=-\frac{\partial p}{\partial x_{i}}}_{\begin{array}{l}
\text { everywhere: } \\
\text { simple boundary conditions }
\end{array}} \Rightarrow \underbrace{\frac{\partial}{\partial t}\left(\rho_{r} u_{i}\right)+\frac{\partial}{\partial x_{j}}\left(\rho_{r} u_{j} u_{i}\right)=-\frac{\partial p}{\partial x_{i}}}_{\begin{array}{c}
\text { within phase-r: } \\
\text { complicated boundary conditions }
\end{array}}
$$

where the two equations differ only in the presence of the subscript $r$ on the density $\rho_{r}$ on the right-hand equation. The cost of this is that while for the left-hand equation one has very simple boundary equations (i.e., at the edges of the box), for the righthand equation one has to specify the boundary conditions between the phases.

From averaging the right-hand equation one obtains[1]

$$
\frac{\partial}{\partial t}\left(f_{r} \rho_{r} \tilde{u}_{i}\right)+\frac{\partial}{\partial x_{j}}\left(f_{r} \rho_{r} u_{j} u_{i}\right)=-f_{r} \frac{\partial p}{\partial x_{i}}-\underbrace{\frac{\partial}{\partial x_{j}}\left(f_{r} T_{r, i j}\right)}_{\begin{array}{c}
\text { Reynold's } \\
\text { stress }
\end{array}}+\underbrace{\frac{\overline{\partial X_{r}}}{\partial x_{i}} p-p_{r} \frac{\partial f_{r}}{\partial x_{i}}}_{\begin{array}{c}
\text { Interfacial } \\
\text { pressures }
\end{array}}
$$

where $\rho_{r}=\bar{\rho}_{r} / f_{r}, X_{r}$ is a characteristic function for phase- $r$ which is 1 in phase- $r$ and 0 elsewhere, and the volume fraction $f_{r}=\overline{X_{r}}$. The first three terms on the left are the analogues to the unaveraged terms in the momentum equation. The last two terms on the right arise arise from the averaging process. The first is analogous to the Reynold's stress which occurs in the usual Reynolds or Favre averaging. In this case it differs in that the velocity fluctuations are with respect to the phase specific mass averaged velocity $\tilde{u}_{r, i}$, and not with respect to the overall mass average velocity $\tilde{u}_{i}$.

The second term arises because the averaging operation was performed over only the phase- $r$ and the average of the pressure gradient within the phase- $r$ is not the 
same as the gradient of the average of the pressure[1]. ${ }^{4}$ This term is typically modeled as drag between the two phases.

Before preceeding with this, it is worthwhile to consider whether anything was obtained from this exercise. The answer to this is an emphatic yes, as the model at this point is able to produce mixing (and demixing). To see this consider a simple problem with a constant pressure gradient $g_{i}=\frac{\partial p_{r}}{\partial x_{i}}$ with the fluids initially at rest. Then the momentum equation initially reduces to

$$
\frac{\partial}{\partial t}\left(f_{r} \rho_{r} \tilde{u}_{i}\right)=-f_{r} g_{i}
$$

From this it is clear that the acceleration of each phase- $r$ will go like $1 / \rho_{r}$. The subsequent velocity separation will result in either mixing or demixing depending on the sign of $g_{i}$ and the initial conditions. Models based on this formalism are also inherently anisotropic and will provide a sort of automatic scale separation if a model is included for the Reynolds stress (i.e., a k-equation, etc.). The costs of this are interfacial pressures to model and phase specific Reynolds stresses.

\section{Drag is not enough}

As was indicated above, the interfacial pressures which appear in the momentum equation are typically modeled as drag between the phases. This is not adequate in the presence of shocks. To see this it is useful to consider the Reynolds stress which appears in the momentum equation

$$
T_{r, i j}=\frac{1}{f_{r}} \overline{X_{r} \rho u_{r, i}^{\prime \prime} u_{r, j}^{\prime \prime}}
$$

From this one can obtain an equation for the energy not resolved in the $\tilde{u}_{r, i}$ if we proceed as follows. First, assume that all the anisotropies are handled by the mean flow variables $\tilde{u}_{r, i}=\overline{\rho_{r} u_{i}} /\left(f_{r} \rho_{r}\right)$. If this is the case, then one can define a phase specific turbulence kinetic energy

$$
k_{r}=\frac{1}{2 \rho_{r}} \sum_{i} T_{r, i i}
$$

If we make the further assumption that all the phases have similar velocity fluctuations $k_{r}$, then one can, without loss of information, sum over the phases to define a total turbulence kinetic energy

$$
k=\frac{1}{\rho} \sum_{r} f_{r} \rho_{r} k_{r}=\frac{1}{2 \rho} \sum_{r, i} f_{r} T_{r, i i}
$$

\footnotetext{
${ }^{4}$ I probably differ from reference [1] on the interpretation of the resulting terms. The form given here is clearer in any case, and likely more correct.
} 
The usual manipulations starting from the momentum equation (along with the assumptions above) eventually produce

$$
\begin{aligned}
& \frac{\partial}{\partial t}(\rho k)+\frac{\partial}{\partial x_{i}}\left(\tilde{u}_{i} \rho k\right)=T_{i n} \sum_{r} f_{r} \frac{\partial \tilde{u}_{r, i}}{\partial x_{i}}+\sum_{r} \frac{\partial}{\partial x_{i}}\left(X_{r} \frac{\rho}{2} u_{r, k}^{\prime \prime} u_{r, i}^{\prime \prime} u_{r, i}^{\prime \prime}\right)
\end{aligned}
$$

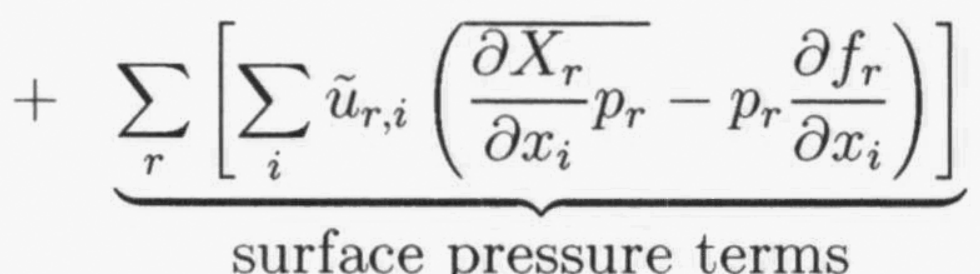

where the first line contains the terms analogous to the single material case and the second line contains the terms arising from the surface pressure terms in the momentum equation. These later terms are obviously not small and have been modeled previously as sources of the energy lost from the velocity slip due to drag and added mass effects $[2,3]$. This is inadequate in the case of a shock interacting with a region containing multiple materials. Further, if a mixed region is initially at rest then $u_{r, i}^{\prime \prime}=0$, and as a consequence $T_{i n}=0$ and similarly for the triple correlation. Hence, only the surface pressure term is available to generate $k$ as is required according to the argument presented in the introduction. This appears plausible since neither the pressure nor the mean flow velocities which appear in this term vanish. The model for this will be called "shock scattering" as was explained in the introduction.

\section{Shock Scattering}

At this point it is clear that there exists a need for a pair of additional terms in the momentum equation and the $k$-equation. I.e., $E_{r, i}$ and $E^{\prime}$ as in

$$
\begin{aligned}
\frac{\partial}{\partial t}\left(f_{r} \rho_{r} \tilde{u}_{i}\right)+\frac{\partial}{\partial x_{j}}\left(f_{r} \rho_{r} u_{j} u_{i}\right) & =-\cdots+E_{r, i} \\
\frac{\partial}{\partial t}(\rho k)+\frac{\partial}{\partial x_{i}}\left(\tilde{u}_{i} \rho k\right) & =\cdots+E^{\prime}
\end{aligned}
$$

Constraints on these two terms arise from the following

1. Conservation of energy and momentum (terms in both the equation for $k$ and the equation for $\tilde{u}_{i}$ are clearly required.)

2. Invariance of the term added to the $k$ equation under changes of the frame of reference.

3. No dimensional constants in either term.

4. Both terms should be independant of the length scale.

5 . Well behaved in the presence of shocks. 


\section{Well behaved in the presence of shocks}

Substantial constraints are placed on the form of $E_{r, i}$ and $E^{\prime}$ (as well as any other term which is used in a mix model) by the requirement that these terms have meaningful behaviour in the presence of discontinuous solutions (i.e., shocks). Recall that there are subtle issues when shocks form in any system of differential equations. The two derivatives $\frac{\partial}{\partial x}$ and $\frac{\partial}{\partial t}$ are not defined (in the usual sense) at a discontinuity. For systems of conservation laws this can be dealt with through the ideas of generalized functions and weak solutions[5]. I.e.,

$$
\begin{aligned}
\text { an equation like: } & \frac{\partial \rho}{\partial t}+\frac{\partial Q(\rho)}{\partial x} & =0 \\
\text { is interpreted to mean: } & \iint_{R}\left\{\rho \frac{\partial \phi}{\partial t}+Q(\rho) \frac{\partial \phi}{\partial x}\right\} & =0
\end{aligned}
$$

where $\phi$ is an arbitrary test function with compact support on $R, \rho$ is some density, and $Q(\rho)$ is some flux. For continuous $\rho$ and $Q(\rho)$ these two equations are equivalent and can be obtained from each other by integration by parts. If $\rho$ or $Q(\rho)$ become discontinuous only the second equation actually has meaning. From these sorts of considerations one also obtains the jump condition $[\rho] \cdot U=[Q(\rho)]$ (where $[X]$ means the change in $X$ across the shock). Because mix models do not generally appear in conservation form ${ }^{5}$, the situation with respect to discontinuous solutions is very murky indeed. Never-the-less, it is clear that the issue of the interpretation of these models in the presence of shocks does not go away just because it has been ignored to date. This issue may become worse in the models than in the Euler equations because of the presence of higher order derivatives and/or products of derivatives.

One can, never-the-less, see that a simple necessary condition exists for these terms to have meaning in the presence of shocks. ${ }^{6}$ If one considers a potential closure $E^{\prime}$ which appears on the right-hand side of some time evolution equation (of $\rho k$ for instance)

$$
\frac{\partial}{\partial t}(\rho k)+\cdots=\cdots+E^{\prime}
$$

then a set of necessary conditions for this term to be meaningful in the presence of

\footnotetext{
${ }^{5}$ This has never happened to my knowledge.

${ }^{6}$ I want to emphasize that this is a necessary but not necessarily sufficient condition for the terms in question to have meaning. If the model were expressed in conservation form, then that would clearly be sufficient. The question as to whether some clever mathematics exists (or can be invented) to give meaning to the model without putting it into conversation form appears to be open.
} 
shocks is ${ }^{7}$

$$
\int_{\text {shock }} E^{\prime} d t=\left\{\begin{array}{l}
\cdot \text { finite } \\
\cdot \text { non-zero } \\
\cdot \text { depends only on the jumps across the shock }
\end{array}\right.
$$

In general one can show that "good" terms "work" by using integration by parts to move the derivatives off the discontinuous functions (so to speak). For "bad" terms no such manipulations appear possible, and it seems to be necessary to introduce a particular continuous form for the step, do the integration explicitly, and then show that the result is not defined (and therefore "does not work") as the step is allowed to become discontinuous. Since the latter is somewhat more transparent, it will be used throughout. Consider a test function

$$
u^{\natural}=u^{\natural}(x+c t)=\left\{\begin{array}{rll}
u_{1} & \text { if } & x+c t<0 \\
u_{1}+(x+c t) \frac{\Delta u}{\Delta} & \text { if } & 0<x+c t<\Delta \\
u_{2}=u_{1}+\Delta u & \text { if } & \Delta<x+c t
\end{array}\right.
$$

i.e., a linear ramp in $u$ which goes from $u_{1}$ to $u_{2}$ over an interval $\Delta$ and which propogates with a velocity $c$. (The superscript $\downarrow$ is used to indicate a discontinuous function.) Then it is trivial to see that in the limit $\Delta \rightarrow 0$ a continuous function times the derivative of a discontinuous function is O.K.

$$
\int_{\text {shock }} f \frac{\partial u^{\natural}}{\partial x} d t \longrightarrow f \frac{\Delta u}{c} \quad \begin{aligned}
& \text { An example of such a term is the pressure } \\
& \text { gradient in the momentum equation. }
\end{aligned}
$$

Several other terms also work. The product of a discontinuous function and the derivative of a discontinuous function is O.K.

$$
\int_{\text {shock }} h^{\natural} \frac{\partial u^{\natural}}{\partial x} d t \longrightarrow\left(h_{1}+\frac{\Delta h}{2}\right) \frac{\Delta u}{c} \quad \begin{aligned}
& \text { Like the } p d V \text { term in the in- } \\
& \text { ternal energy equation. }
\end{aligned}
$$

The product of a continuous function and the n'th derivative of a discontinuous function also works

$$
\int_{\text {shock }} h \frac{\partial^{n} u^{\natural}}{\partial x^{n}} d t \longrightarrow(-1)^{n-1} \frac{\partial^{n-1} h}{\partial x^{n-1}} f \frac{\Delta u}{c}
$$

This last case follows, roughly speaking, because one can take arbitrary derivatives of $\delta$-functions. (Although this might be hard to actually make work in a real code ...)

\section{There are also at least a couple of terms which do not work.}

The product of the derivatives of two discontinuous functions

$$
\int_{\text {shock }} \frac{\partial g^{\natural}}{\partial x} \frac{\partial u^{\natural}}{\partial x} d t \longrightarrow \frac{\Delta g \cdot \Delta u}{c \cdot \Delta} \longrightarrow \infty
$$

\footnotetext{
${ }^{7}$ The requirement that the result be non-zero is not strictly necessary, zero being a perfectly good number. However, such a term is clearly of no use in modeling the shock-mix interaction.
} 
and the product of a discontinuous function and the second derivative of a discontinuous function

$$
\int_{\text {shock }} g^{\natural} \frac{\partial^{2} u^{\natural}}{\partial x^{2}} d t \longrightarrow-\frac{\Delta g \cdot \Delta u}{c \cdot \Delta} \longrightarrow-\infty
$$

are both undefined. This is roughly the equivalent of saying that there is no meaning to multiplying $\delta$-functions. (Note that these two cases are also related by an integration by parts.) Note also that the first of these rules out the use of $\frac{\partial \rho}{\partial x} \cdot \frac{\partial p}{\partial x}$.

\section{Some terms are zero.}

These are a couple of almost trivial examples. The integral over a shock of either a continuous or discontinuous function vanishes.

$$
\int_{\text {shock }} f d t=\int_{\text {shock }} g^{\natural} d t=0
$$

The point here being that one requires a certain degree of singular behavior out of the term in order to get a non-zero contribution as the width of the shock goes to zero. More complicated terms can also be zero. For instance

$$
\begin{aligned}
\int_{\text {shock }} \frac{\partial}{\partial x}\left(g^{\natural} \frac{\partial u^{\natural}}{\partial x}\right) d t & =\int_{\text {shock }} \frac{\partial g^{\natural}}{\partial x} \frac{\partial u^{\natural}}{\partial x}+\int_{\text {shock }} g^{\natural} \frac{\partial^{2} u^{\natural}}{\partial x^{2}} \\
& =\frac{\Delta g \cdot \Delta u}{c \cdot \Delta}-\frac{\Delta g \cdot \Delta u}{c \cdot \Delta}=0
\end{aligned}
$$

Figure 2 shows a rather schematic representation of this potential source term. The point to appreciate is that one might have difficulties integrating this numerically and actually getting zero.

\section{Terms which "do not work" are commonly used in turbulence modeling.}

Some of the terms indicated above which "do not work" are used in turbulence modeling in cases where there are typically no shocks. An example of such a situation is a common closure for the Reynolds stress[7].

$$
\overline{\rho u_{i}^{\prime \prime} u_{i}^{\prime \prime}}=T=-\underbrace{\mu_{T} \frac{4}{3} \frac{\partial u}{\partial x}}_{\mathrm{A}}+\underbrace{\frac{2}{3} \rho k}_{\mathrm{B}}, \quad \mu_{T}=\rho \sqrt{k} l
$$

The labels A and B will be used in the discussion below as these two parts of the closure behave in a very different manner in the presence of a shock. This term appears in both the momentum equation and in the equation for $k$, acting as a sort of 


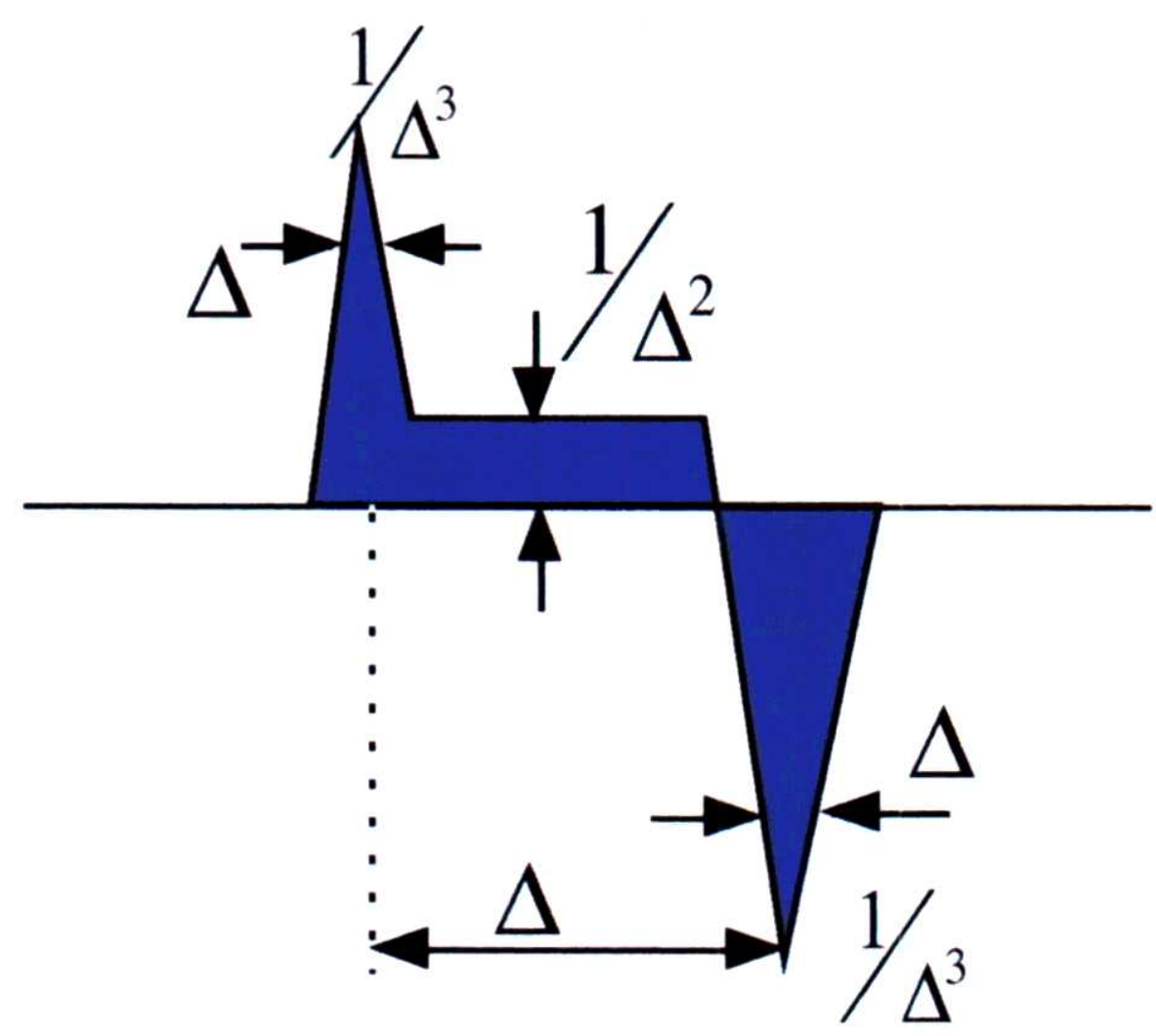

Figure 2: The figure shows the complex singular behaviour of the term in eqn.28. Labels show proportionality to $\Delta$. This term, never-the-less, integrates to zero.

turbulent pressure which can move energy between the mean flow and the unresolved scales $k$.

$$
\begin{gathered}
\frac{\partial}{\partial t}(\rho u)+\cdots=\cdots-\frac{\partial T}{\partial x}=\cdots \underbrace{\frac{\partial}{\partial x}\left(\mu_{T} \frac{4}{3} \frac{\partial u}{\partial x}\right)}_{\mathrm{A}}-\underbrace{\frac{2}{3} \frac{\partial}{\partial x}(\rho k)}_{\mathrm{B}} \\
\frac{\partial}{\partial t}(\rho k)+\cdots=\cdots-T \frac{\partial u}{\partial x}=\cdots \underbrace{\mu_{T} \frac{4}{3}\left(\frac{\partial u}{\partial x}\right)^{2}}_{\mathrm{A}}-\underbrace{\frac{2}{3} \rho k \frac{\partial u}{\partial x}}_{\mathrm{B}}
\end{gathered}
$$

By the criteria given above the B terms "work." The A terms do not. The A term in the $k$-equation contains the product of two derivatives of a discontinuous function, and will make an undefined contribution to $k$.

$$
\int_{\text {shock }} \mu_{T} \frac{4}{3}\left(\frac{\partial u}{\partial x}\right)^{2} d t=\rho \sqrt{k} l \cdot \frac{4}{3} \cdot \frac{(\Delta u)^{2}}{c \cdot \Delta} \propto \frac{1}{\text { zone size! }}
$$

The corresponding A term in the momentum equation has no net effect on the momentum, but because it acts to accelerate and decelerate the flow at times at which the flow velocity is very different (recall the discontinuous change in $u$ ), it can neverthe-less change the energy in the mean flow.

In any code, of course, shocks always have a finite width so that in practice "bad terms" as identified here will have large but finite effects due to shocks. In an Euler code (one with no explicit viscosity) these effects will be proportional to inverse powers 
of the zone size (since shocks are typically spread over a few zones by artificial viscous effects of one sort or another). This means that models with such terms will prevent the code from reaching a converged answer as the zone size is reduced. In a code with explicit viscous effects, "bad terms" will produce effects which depend upon the viscosity. As shock interactions are generally thought to be independent of the actual viscosity and thermal conductivity which determine the width of the shock[6], this would appear to be the wrong physics.

These observations are not entirely new. At least one author[8] understood that some of these terms get too big, and that limiters must be used. While anecdotal evidence suggests that the use of limiters may be more wide spread and not generally reported in the literature [4, 9], it is not clear that the fundamentally undefined character of these terms has been appreciated. To put this another way, the difficulty here goes beyond the idea of realizability constraints. [7]

Lastly, one should ask if there are any ways around the difficuties described in this section. If the model were to act upon the mean flow variables so as to induce a correct shock width (in the average sense), then all the "bad" terms would be defined. Such an interaction is expected in some sense, of course, because the Reynolds stress term in the momentum equation can have the form of a viscosity. The usual closure will presumably not work in the present case because it is (at best) a shock-turbulence interaction and not a shock-mix interaction. Even with a closure which induces the correct shock width, it might be a difficult task to get overall reasonable behaviour. I.e., it is not clear that a "bad" term with a value of $\Delta$ proportional to the average shock width induced by the passage through a mix material would give reasonable behaviour. Such a model would also require that the resolution in the simulation be adequate to resolve the shock width.

\section{Dimensional Analysis}

If the new term in the $k$-equaiton is to not have a dimensionful constant out front, and if it does not depend on the length scale $l$ in the problem, then it is straightforward to show that

$$
\frac{\partial}{\partial t}(\rho k)+\cdots=C \cdot \rho^{\alpha} u^{1+2 \alpha} p^{1-\alpha} \frac{\partial}{\partial x}
$$

where the derivative $\frac{\partial}{\partial x}$ could be applied to any combination of the powers of $\rho, u$, and $p$. At this point $\alpha$ remains a free parameter.

\section{Invariance}

It is well known that $E^{\prime}$ must be invariant under a change in coordinate systems. The term $u^{1+2 \alpha}$ above might break such an invariance. This can be "fixed" either by 
taking the exponent of $u$ to be zero so that $\alpha=-1 / 2$, or by taking the exponent to be one so that $\alpha=0$ and letting the derivative operate on it. These two cases result in

$$
\frac{\partial}{\partial t}+\cdots=C \cdot \sqrt{\frac{p}{\rho}} \frac{\partial p}{\partial x} \quad \text { or } \quad \frac{\partial}{\partial t}+\cdots=C \cdot p \frac{\partial u}{\partial x}
$$

The left one being roughy a pressure gradient times a sound speed and the right one a $p \delta V$ work term.

\section{Conservation of Energy and Momentum}

Lastly, the need to conserve momentum and energy forces the selection of the righthand term. The total energy equation is obtained by multiplying the momentum equation in two forms by $u$ and adding these to the equations for $k$ and internal energy. Conservation is achieved if the combined term $u \cdot E_{r, i}+E^{\prime}$ either vanishes or forms a total divergence. The first of these would correspond to a purely local exchange of energy between the mean flow and $k$. The second case allows for energy transport as well as exchange. The first would require an inverse power of $u$ in the term in the momentum equation and, therefore, appears to be unreasonable. Hence the only possible forms for $E_{r, i}$ and $E^{\prime}$ appear to be

$$
E_{r, i}=C \cdot \frac{\partial p}{\partial x} \quad \text { and } \quad E^{\prime}=C \cdot p \frac{\partial u}{\partial x}
$$

which is exactly the same form as the $p \delta V$ work terms which exchange energy between the mean flow and internal energy. Note also that the term in the momentum equation must be a divergence so as to conserve momentum.

\section{Non-dimensional factors}

At this point we are required to specify the form of the non-dimensional factor $C$. As was discussed above, this term must be sensitive to 1) the presence of a mixed region, and 2) the presence of a shock. Toward this end I first introduce

$$
H_{i}=F_{i} \sum_{r} f_{r} H_{r, i}
$$

which is the product of two factors. The first $F_{i}$ will be sensitive to the presence of a shock. The second (the sum) is sensitive to the presence of a mixed region. Specifically

$$
H_{r, i}=\sum_{s \neq r} f_{s} \frac{\left|\rho_{r}-\rho_{s}\right|}{\rho_{r}+\rho_{s}}
$$




\begin{tabular}{|l|l|l|}
\hline$c_{1}$ & $17.25-5.25 \cdot A t$ & drag \\
\hline$c_{2}$ & 0.75 & turblent length scale w.r.t. 1 \\
\hline$c_{4}$ & 0.09 & dissipation of turbulent KE \\
\hline$c_{5}$ & 2.0 & mass diffusion \\
\hline$c_{7}$ & 1.0 & turbulent KE diffusion \\
\hline$c_{8}$ & 2.0 & length scale diffusion \\
\hline$c_{10}$ & 4.0 & shock scattering \\
\hline$c_{11}$ & 0.005 & mix trigger \\
\hline$c_{s s s}$ & $0.0,0.3$ & compression / expansion scale factors \\
\hline$c_{l s c}$ & 1.0 & length scale source \\
\hline$c_{l s c 2}$ & 1.0 & length scale due to diffusion \\
\hline
\end{tabular}

Table 1: Parameters used in the simulations described below. As described in the text, some have been picked to fit the data, others are from the literature $[2,3]$.

so that $\sum_{r} f_{r} H_{r, i}$ is just a weighted sum of Atwood numbers. The $F_{i}$ term is the $n$ 'th root of a product over phases

$$
F_{i}=\left[\prod_{r} F_{r, i}\right]^{\frac{1}{n}} \text { where } F_{r, i}=\left\{\begin{array}{ccc}
0 & \text { iff } & \delta u_{i}<\delta u_{l} \\
0.5\left(\frac{\delta u_{i}-\delta u_{l}}{\delta \delta u}\right)^{c_{13 c}} & \text { iff } & \delta u_{l}<\delta u_{i}<\delta u_{o} \\
0.5\left(\frac{\delta u_{h}-\delta u_{i}}{\delta \delta u}\right)^{c_{13 c}} & \text { iff } & \delta u_{0}<\delta u_{i}<\delta u_{h} \\
1 & \text { iff } & \delta u_{h}<\delta u_{i}
\end{array}\right.
$$

and $\delta u_{i}=u_{i-\frac{1}{2}}-u_{i+\frac{1}{2}}, \delta u_{0}=c_{13 a}, \delta \delta u=c_{13 a} \cdot c_{13 b}, \delta u_{l}=\delta u_{0}-\delta \delta u$, and $\delta u_{h}=$ $\delta u_{0}-\delta \delta u$. While the algebra is a little tedious, the function $F_{i}$ simply implements a smooth transition from zero to one as the relative velocity between two zone edges goes from less than $\delta u_{0}=c_{13 a}$ to greater than $\delta u_{0}$. The final result is

$$
\begin{array}{r}
\frac{\partial}{\partial t}\left(f_{r} \rho_{r} \tilde{u}_{r, i}\right)+\cdots=\cdots c_{10} \frac{\partial}{\partial x_{i}}\left(f_{r} p_{r} H_{i}\right) \\
\frac{\partial}{\partial t}(\rho k)+\cdots=\cdots c_{10} \sum_{r, i} f_{r} p_{r} H_{i} \frac{\partial \tilde{u}_{r, i}}{\partial x_{i}}
\end{array}
$$

where $c_{10}$ is a constant. Note that $H_{i}$ must appear inside one derivative and outside the other so that the resulting combined term in the total energy equation is still a total divergence.

\section{Length scale sources}

As will be discussed below, changes were also required to the length scale source terms in order to match the data. The dilatation term was given separate scale factors for 
expansion and compression, and an additional source which allows purely turbulent mix to generate its own length scale has been added.

In addition to the above, it was found necessary to limit the development of additional velocity separation during shocks so as to prevent a sort of double counting from occurring. This is accomplished by interpolating between the pressure gradient given above, and the form which occurs in the single fluid case. This interpolation is based on $F_{i}$ defined above to identify shocks.

\section{Implementation}

The model has been implemented as a 1-D Lagragian hydrocode. The final form is as follows.

$$
\begin{aligned}
\frac{\partial}{\partial t}\left(A f_{r} \rho_{r}\right)+\frac{\partial}{\partial x}\left(A f_{r} \rho_{r} \tilde{u}_{r}\right) & =0 \\
\frac{\partial}{\partial t}\left(A f_{r} \rho_{r} \tilde{u}_{r}\right)+\frac{\partial}{\partial x}\left(A f_{r} \rho_{r} \tilde{u}_{r} \tilde{u}_{r}\right) & =A f_{r} \frac{\partial p}{\partial x}+\sum_{\text {shock scattering }}^{\text {Reynolds stress }} \frac{\partial T}{\partial x}+A \sum_{s} D_{r s} \\
& +c_{10} A \frac{\partial}{\partial x}\left(p \cdot f_{r} H\right)+A f_{r} S_{u, r} \\
\frac{\partial}{\partial t}\left(A f_{r} \rho_{r} e_{r}\right)+\frac{\partial}{\partial x}\left(A f_{r} \rho_{r} e_{r} \tilde{u}_{r}\right) & =-h_{r} p \frac{\partial}{\partial x}(A \bar{u})+A f_{r} \epsilon+A f_{r} S_{e, r} \\
\frac{\partial}{\partial t}(A \rho k)+\frac{\partial}{\partial x}(A \rho k \tilde{u}) & =\frac{-T \frac{\partial \bar{u}}{\partial x}-\frac{\partial}{\partial x}\left(A \rho D_{k} \frac{\partial k}{\partial x}\right)-\epsilon}{\text { Reynolds stress }} \\
& \left.+\sum_{r<s} f_{r} \tilde{w}_{r} \frac{\partial p}{\partial x}+\tilde{u}_{s}-\tilde{u}_{r}\right)\left(D_{r s}-M_{r s} f_{r} p H \frac{\partial \tilde{u}_{r}}{\partial x}\right. \\
\frac{\partial l}{\partial t}+\left(\bar{u}+u_{l}\right) \frac{\partial l}{\partial x} & =-\frac{1}{A} \frac{\partial}{\partial x}\left[A D_{l} \frac{\partial l}{\partial x}+c_{s s} \frac{\partial \bar{u} l}{\partial x} l+S_{l}+S_{l 2}\right.
\end{aligned}
$$

where

$$
\begin{aligned}
S_{l}=c_{l s c} \cdot \frac{\sum_{r>s} f_{r} f_{s}\left(\frac{2 \rho_{r s}}{\rho_{r}+\rho_{s}}\right)^{\frac{1}{2}}\left(\tilde{u}_{r}-\tilde{u}_{s}\right)}{\sum_{r>s} f_{r} f_{s}} & \text { and } & S_{l 2}=c_{l s c 2} \cdot \frac{\sum_{r>s} f_{r} f_{s}\left(\frac{2 \rho_{r s}}{\rho_{r}+\rho_{s}}\right)^{\frac{1}{2}}\left(\tilde{u}_{r}-\tilde{u}_{s}\right)}{\sum_{r>s} f_{r} f_{s}} \\
u_{l}=\frac{\sum_{r>s} f_{r} f_{s}\left(f_{r}-f_{s}\right)\left(\tilde{u}_{s}-\tilde{u}_{r}\right)}{\sum_{r>s} f_{r} f_{s}} & & \bar{u}=\sum_{r} f_{r} \tilde{u}_{r} \\
D_{r s}=-c_{1} \frac{\rho_{r s} f_{r} f_{s}}{l}\left|\Delta_{r s}\right| \Delta_{r s} & \text { with } & \Delta_{r s}=\left(\tilde{u}_{r}-\tilde{w}_{r}\right)-\left(\tilde{u}_{s}-\tilde{w}_{s}\right)
\end{aligned}
$$




$$
\begin{array}{lll}
\tilde{w}_{r}=-\frac{D}{f_{r} \rho_{r}} \frac{\partial}{\partial x}\left(f_{r} \rho_{r}\right) \quad \text { with } \quad D=c_{5} \sqrt{k} l_{t}, l_{t}=c_{2} l \\
\text { Reynolds stress } \\
T=-\mu_{T} \frac{4}{3} \frac{\partial \tilde{u}}{\partial x}+\frac{2}{3} \rho k & \text { limited by } & \left.0<T<\frac{5}{4} \rho k \quad \text { (see }[8]\right)
\end{array}
$$

As has been indicated previously, this is derived from the work of Youngs[2, 3, 4]. Deviations from that work are indicated in the boxes. They fall into four groups.

- Shock scattering terms (proportional to $c_{10}$ ) appear in the momentum and $k$ equations.

- Reynolds stress terms of a different sort also appear in these two equations. Note the need for a limiter on $T$.

- The definition of $\bar{u}$ as it appears in the $p \delta V$ work term in the energy equation has been changed. This allows a "bad" term to be deleted from the $k$-equation as discussed above.

- Two length scale source terms in the length scale equation are changed.

The parameter set used in the simulations is given in Table 1.

\section{Comparison to Experiments}

This effort was originally motivated by the known inability of the model to handle heavy to light RMI. This section compares the model to two experiments. The first is a heavy to light reshock experiment in which improved behaviour is observed. Since the changes (particularly those to the length scale sources) will change the behaviour of the model in general, a comparison is made to an acceleration / deceleration "rocket rig" experiment. A reasonable agreement with the data is obtained, with the two experiments together substantially constraining the model.

\section{Reshock Experiment}

The first comparison is to the reshock experiment of Vetter and Sturdevant[10]. This is an air- $\mathrm{SF}_{6}$ Mach 1.5 shock tube experiment. The initial interface is formed by a flat membrane. The incident shock brakes the membrane and then is reflected from the end wall $(62 \mathrm{~cm}$ from the inital interface location). This reflection (a heavy to light shock) accelerates the interface approximately $3.4 \mathrm{msec}$. after the initial shock and is observed to cause a substantial growth in the mixing rate. 


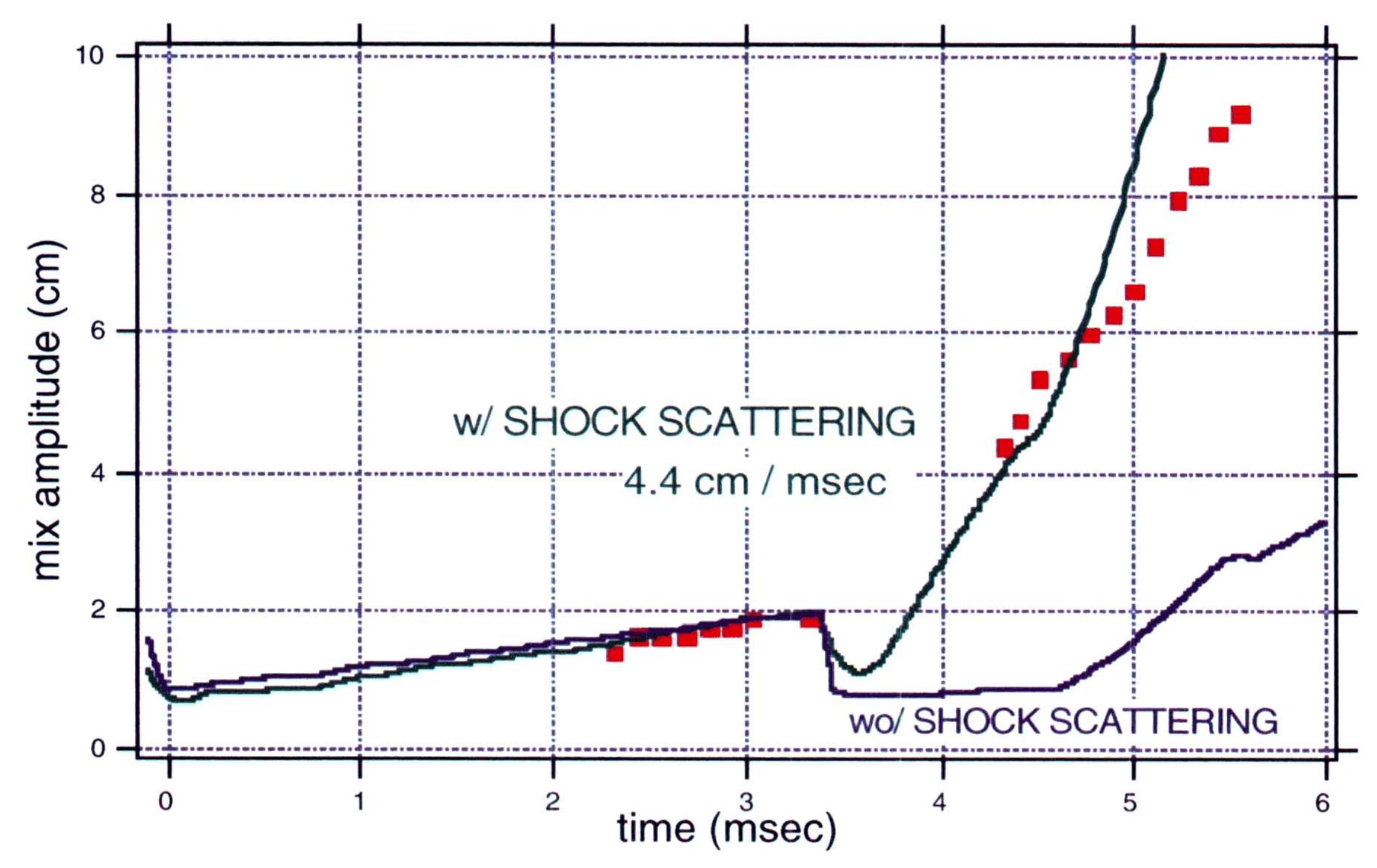

Figure 3: The figure shows a comparison between the measured and modeled values of the total mix width in the reshock experiment. The red points are from the experiment. The blue and green solid lines show the results of the model with and without the shock scattering. Reasonable agreement is obtained up until the arrival of the reflected rarefaction at about $4.5 \mathrm{msec}$.

The simulation of this experiment is carried out with ideal gas equations-of-state $\left(\gamma=1.402\right.$ for air and $\gamma=1.0935$ for $\left.\mathrm{SF}_{6}\right)$. The initial value of the length scale $l$ in the model was adjusted so that the width of the mix region measured at 3 msec. in the experiment was reproduced by the model. The results are compared to the experiment in Fig. 3. Good agreement is obtained up until the arrival of a reflected rarefaction at $4.5 \mathrm{msec}$. The behaviour of the unmodified model is also shown. ${ }^{8}$

\section{Acceleration / Deceleration Experiment}

The second comparison is to the acceleration/deceleration experiment of Smeeton and Youngs[11]. This was an immiscible fluid "rocket rig" experiment carried out at AWE in which the tank first experienced a constant acceleration, followed by a coast, followed by a more or less constant deceleration, followed by another coast. The

\footnotetext{
${ }^{8} \mathrm{~A}$ reasonable effort was made to tune the unmodified model to reproduce the data. While this was not successful, it is difficult to really prove that is not possible.
} 


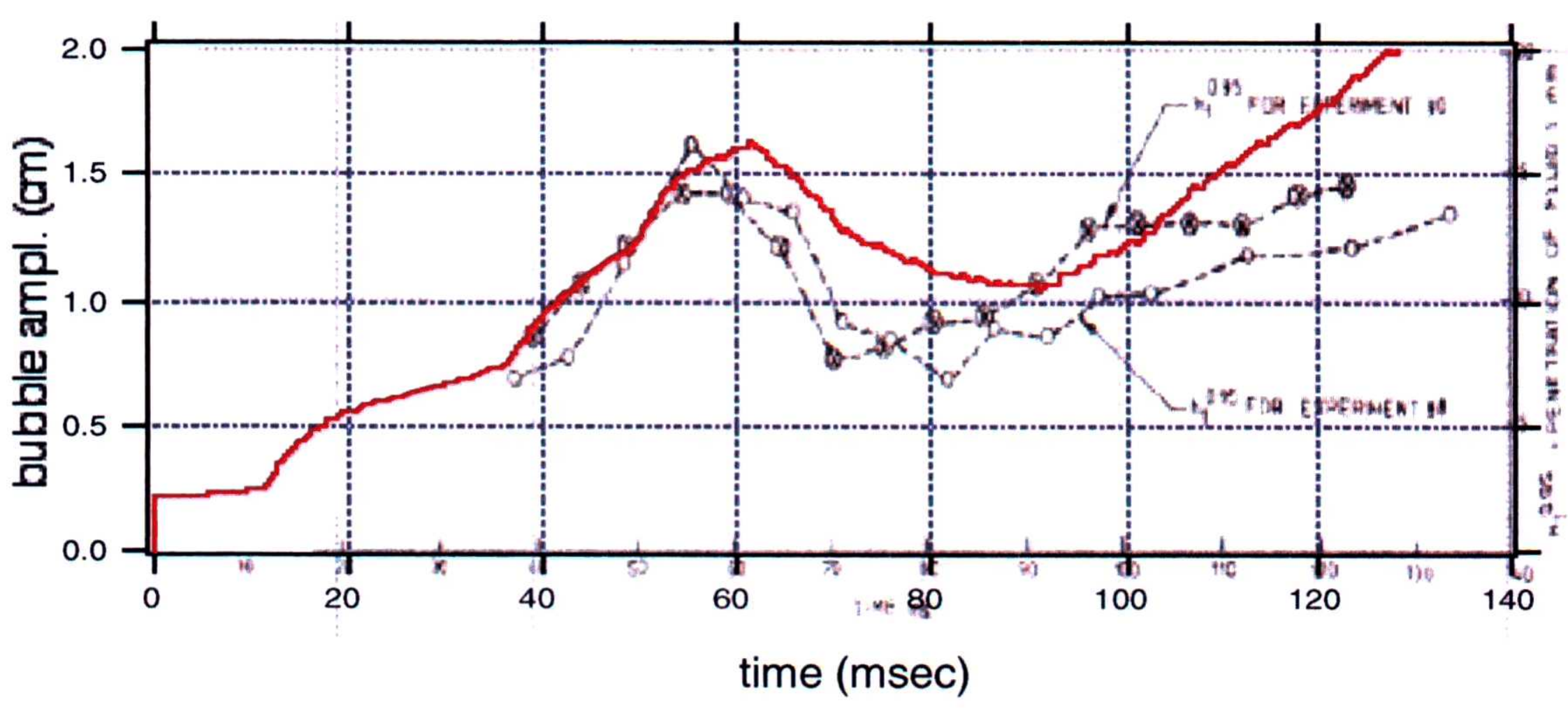

Figure 4: The figure shows a comparison between the measured and modeled values of the bubble amplitude. The model results are shown in red. Reasonable agreement is obtained.

\section{Acknowledgements}

I wish to thank Harry Robie and Oleg Schilling for useful conversations. I also wish to thank David Youngs for invaluable information on implementing multiphase flow mix models.

This work was performed under the auspices of the U.S. Department of Energy by the University of California Lawrence Livermore National Laboratory under contract No. W-7405-Eng-48.

\section{References}

[1] D. A. Drew and S. L. Passman, Theory of Multicomponent Fluids, (Springer, New York, NY, 1999).

[2] David L. Youngs, "Numerical Simulation of Turbulent Mixing by Rayleigh-Taylor Instability," Physica D37 270-287 (1989).

[3] David L. Youngs, "Numerical simulation of mixing by Rayleigh-Taylor and Richtmyer-Meshkov instabilities," Laser and Part. Beams 12 725-750 (1994).

[4] Youngs, D. L., AWE Aldermaston, private communications.

[5] G. B. Whitham, Linear and Nonlinear Waves, (Wiley, New York, 1999). 
[6] Gabi, B., Ozer, I., Elperin, T. Eds., Handbook of Shock Waves, (Acedemic Press, San Diego, 2001).

[7] David C. Wilcox, Turbulence Modeling for CFD 2nd Ed., (DCW Industries, La Cañada, CA, 1998)

[8] S. Gauthier and M. Bonnet, "A k- $\epsilon$ model for turbulent mixing in shock-tube flows induced by Rayleigh-Taylor instability," Phys. Fluids A 2 (1990) 1685-1694.

[9] Schilling, O., Lawrence Livermore National Laboratory, private communication (2003).

[10] Vetter and Sturtevant, "Experiments on the Richtmyer-Meshkov instability of an Air / SF 6 interface," Shock Waves, 4, 247-252 (1994).

[11] Smeeton, V. S. and Youngs, D. L., Experimental Investigation of Turbulent Mixing by Rayleigh-Taylor Instability - Part 3, Atomic Weapons Establishment Aldermaston, AWE Report No. O 35/87 (1987). 